Cite this: Soft Matter, 2013, 9, 8761

\title{
Translational and rotational dynamics in suspensions of magnetic nanorods $\uparrow$
}

\author{
Carlos E. Alvarez* and Sabine H. L. Klapp \\ Using computer simulations we investigate the translational and rotational diffusion of dilute suspensions \\ of magnetic nanorods with and without a (homogeneous) external magnetic field. The magnetic rods are \\ represented as spherocylinders with a longitudinal point dipole at their center and length-to-breadth ratios \\ $L I D=3$ or $L I D=9$. In the absence of a field, the rods tend to form compact clusters with antiparallel \\ ordering and thus behave very differently to dipolar spheres $(L I D=0)$, which tend to form head-to-tail \\ chains. Furthermore, for rod-like particles the external field tends to destabilize rather than to support \\ cluster formation. We show that these differences in the aggregation behavior have profound \\ consequences not only in static material properties such as the field-induced magnetization and the \\ zero-frequency susceptibility, but also in the dynamics. In particular, for magnetic rods the translational \\ diffusion constant parallel to the field is larger than the perpendicular one, in contrast to the behavior \\ observed for magnetic spheres. Moreover, the rod-like character greatly affects the shape and the \\ density dependence of the single-particle and collective dipole-dipole time correlation functions and \\ their counterparts in the frequency domain.
}

Received 3rd June 2013

Accepted 22nd July 2013

DOI: $10.1039 / c 3 s m 51549 d$

www.rsc.org/softmatter

established tools to study models which are too complicated to be treated analytically, but that remain simple enough to hopefully give us some insight into the mechanisms underlying the behavior of magnetic fluid systems. In this spirit, we present here a Molecular Dynamics (MD) simulation study of a model magnetic nanorod system composed of spherocylinders with central, longitudinal point dipoles. Our aim is to explore the interplay between structure formation and translational and rotational dynamics, both in globally isotropic systems and in the presence of an external magnetic field. Indeed, external fields play an important role in many experiments (see e.g. ref. 13), and are known to strongly influence the structure formation already in suspensions of magnetic spheres. ${ }^{3}$

Dipolar spherocylinders (and related models involving prolate spheroids or prolate Gay-Berne particles, plus an internal point dipole) have been studied widely both by theory and by computer simulations (see, e.g., ref. 14-18), including studies based on MD simulations. ${ }^{19,20}$ However, most of these studies focus on the role of the dipolar rod-rod interactions on the formation of liquid-crystalline (e.g., nematic or smectic) mesophases, which for non-dipolar rods occur for aspect ratios $L / D>3.7 .{ }^{21}$ Therefore, most of these earlier studies concentrate on dense systems characterized by volume fractions $\eta>0.1$. In contrast, typical ferrofluid suspensions have volume fractions of magnetic particles ranging between $\eta=0.01$ and $0.2,,^{10,22,23}$ indicating that for real magnetic nanorod suspensions the lowdensity regime is of particularly high relevance. The present study therefore concentrates on rather dilute systems. Moreover, we restrict our simulations to low and moderate magnetic

Institut für Theoretische Physik, TU Berlin, Hardenbergstraße 36, D-10623 Berlin, Germany.E-mail: carlose.alvarez@uniandes.edu.co

$\dagger$ Electronic supplementary information (ESI) available. See DOI: $10.1039 / \mathrm{c} 3 \mathrm{sm} 51549 \mathrm{~d}$ 
coupling strength. Within this regime, we focus on diffusive properties with and without a magnetic field, and on the magnetic response.

As this is, to our knowledge, the first study addressing the dynamics of diluted magnetic nanorods based on computer simulations, and due to the large parameter space that such systems present, we have decided to leave aside, as a first step, hydrodynamic interactions (HI). The latter may play an important role in experimental systems ${ }^{24}$ but add significant computational burden. Moreover, neglecting HI as a starting point allows us to better understand the sole effect of dipolar and steric interactions on the dynamics, a question, which is also relevant for molecular systems of elongated dipolar particles.

The rest of the paper is organized as follows. In Section 2.1 we specify the dipolar rod model used in the simulations and the interaction potential between the particles. Details of the molecular dynamics simulations are presented in Section 2.2. Section 3.1 begins with a discussion of the structure formation in the dipolar sphere and spherocylinder systems, followed by the results for the orientational order of the systems presented in Section 3.2. Sections 3.3 and 3.4 are dedicated to the translational and rotational dynamics of the systems, respectively, and in Section 3.5 we present the results for the frequency dependent susceptibility. Finally, Section 4 summarizes our results.

\section{Model and simulations}

\subsection{Model}

We study monodisperse systems composed of $N$ soft magnetic rods, where each particle is represented by a cylinder of length $L$ and diameter $D$ with two hemispherical caps of diameter $D$ (spherocylinder), plus a longitudinal point dipole $\boldsymbol{m}$ at its center (see Fig. 1). The short range, repulsive, soft spherocylinder potential is given by

$$
U_{\mathrm{sr}}=\left\{\begin{array}{ll}
4 \varepsilon\left[\left(\frac{D}{d}\right)^{12}-\left(\frac{D}{d}\right)^{6}\right]+\varepsilon & \text { if } \quad d \leq 2^{1 / 6} D \\
0 & \text { if } \quad d>2^{1 / 6} D
\end{array},\right.
$$

where $d$ is the closest distance between the surfaces of the two spherocylinders and $\varepsilon$ is an energy parameter. Eqn (1) reduces to a truncated-shifted Lennard-Jones potential when $L=0$, where $D$ is the diameter of the sphere and $r=d+D$ is the distance between the centers of the spheres.

In addition, two particles interact via long range dipoledipole interactions given by

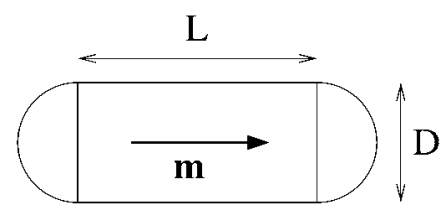

Fig. 1 Sketch of a magnetic dipolar spherocylinder.

$$
U_{\mathrm{dip}}=\frac{\boldsymbol{m}_{i} \cdot \boldsymbol{m}_{j}}{\boldsymbol{r}_{i j}{ }^{3}}-3 \frac{\left(\boldsymbol{m}_{i} \cdot \boldsymbol{r}_{i j}\right)\left(\boldsymbol{m}_{j} \cdot \boldsymbol{r}_{i j}\right)}{\boldsymbol{r}_{i j}{ }^{5}},
$$

in which $\boldsymbol{m}_{i}$ is the dipole moment of particle $i$ and $\boldsymbol{r}_{i j}=\boldsymbol{r}_{i}-\boldsymbol{r}_{j}$ is the separation vector between particles $i$ and $j$.

The total Hamiltonian of the system, including kinetic and potential parts, is then given by

$$
\begin{aligned}
H= & \sum_{i} \frac{1}{2}\left(\nu_{i} u_{i}{ }^{2}+\omega_{i}{ }^{T} \overline{\boldsymbol{I}}_{i} \boldsymbol{\omega}_{i}\right)+\sum_{i j}\left(U_{\mathrm{sr}}\left(\boldsymbol{r}_{i j}, \Omega_{i}, \Omega_{j}\right)\right. \\
& \left.+U_{\mathrm{dip}}\left(\boldsymbol{r}_{i j}, \Omega_{i}, \Omega_{j}\right)\right)+\sum_{i} \boldsymbol{m}_{i} \cdot \boldsymbol{H}
\end{aligned}
$$

where $\nu_{i}$ and $\overline{\boldsymbol{I}}_{i}$ are the mass and inertia tensor of particle $i, \Omega_{i}$ is its orientation, $u_{i}$ and $\omega_{i}$ are its speed and angular velocity respectively, and $\boldsymbol{H}$ is the external field.

Since we are aiming to describe a bulk system, we use periodic boundary conditions (PBC) in three dimensions. To this end the dipolar interactions are handled via the (three dimensional) Ewald summation. The dipolar interaction takes the form ${ }^{25,26}$

$$
\begin{aligned}
U_{\text {dip }}= & \sum_{\boldsymbol{n}}^{\prime}\left[\frac{1}{2} \sum_{i} \sum_{j \neq i}\left(\boldsymbol{m}_{i} \cdot \boldsymbol{m}_{j}\right) A-\left(\boldsymbol{m}_{i} \cdot \tilde{\boldsymbol{r}}_{i j}\right)\left(\boldsymbol{m}_{j} \cdot \tilde{\boldsymbol{r}}_{i j}\right) B\right] \\
& +\frac{1}{V} \sum_{\boldsymbol{k} \neq 0} \frac{4 \pi}{k^{2}} \mathrm{e}^{-k^{2} / 4 \alpha^{2}} \frac{1}{2} \sum_{i} \sum_{j \neq i}\left(\boldsymbol{m}_{i} \cdot \boldsymbol{k}\right)\left(\boldsymbol{m}_{j} \cdot \boldsymbol{k}\right) \mathrm{e}^{-i \boldsymbol{k} \cdot \boldsymbol{r}_{i j}}
\end{aligned}
$$

In eqn (4) $\boldsymbol{n}=\left(n_{x}, n_{y}, n_{z}\right)$ where $n_{\alpha}$ can be 0 or a positive integer, and $\tilde{\boldsymbol{r}}_{i j}=\boldsymbol{r}_{i}-\boldsymbol{r}_{j}+\overline{\boldsymbol{h}} \boldsymbol{n}$ with

$$
\overline{\boldsymbol{h}}=\left(\begin{array}{ccc}
L_{x} & 0 & 0 \\
0 & L_{y} & 0 \\
0 & 0 & L_{z}
\end{array}\right),
$$

where $L_{x}=L_{y}=L_{z}$ are the dimensions of the simulation box. Furthermore

$$
\begin{gathered}
A=\frac{\operatorname{erfc}\left(\alpha \tilde{r}_{i j}\right)}{\tilde{r}_{i j}{ }^{3}}+\frac{2 \alpha}{\sqrt{\pi}} \frac{\mathrm{e}^{-\alpha^{2} \tilde{r}_{i j}{ }^{2}}}{\tilde{r}_{i j}{ }^{2}} \\
B=-\nabla A \cdot \frac{\tilde{\boldsymbol{r}}_{i j}}{\tilde{r}_{i j}{ }^{2}} .
\end{gathered}
$$

The wave vectors are given by $\boldsymbol{k}=2 \pi \overline{\boldsymbol{h}}^{-1} \boldsymbol{n}$. The forces and torques are obtained from the potentials (1) and (4) via the relations $\boldsymbol{F}_{i}=-\nabla_{i} U$ and $\tau_{i}=-\boldsymbol{m}_{i} \times \partial U / \partial \boldsymbol{m}_{i}$.

To compute the dipole-dipole interactions we stick to the minimum image convention $(\boldsymbol{n}=0$ in the real part of the summation), and use values of $\alpha$ in the range $5 \leqq \alpha L_{i} \lesssim 7.5$ and maximum wave vector values $\left(k_{\max }=\left|\boldsymbol{k}_{\max }\right|\right)$ in the range $5<k_{\max }<9$, depending on the dimensions $\left(L_{i}\right)$ of the system.

\subsection{Simulations}

We performed molecular dynamics (MD) simulations of dipolar spherocylinder systems composed of $500 \leq N \leq 1500$ particles in the canonical $(N, V, T)$ ensemble, within a cubic simulation box under PBC. We used the leap-frog Verlet method to solve numerically the equations of motion with a time step $10^{-4} \leq$ $\Delta t^{*} \leq 10^{-3}$ (for the definition of $t^{*}$ see below). Smaller time 
steps were used for longer rods to reduce the probability of occurrence of unphysical overlaps that may introduce artifacts in the dynamics of the system. In order to sample from the canonical ensemble, two independent Hoover-Langevin thermostats $^{27}$ for the translational and rotational degrees of freedom respectively were used.

In the present study we focus on the system's behavior at low densities below the onset of a nematic (or otherwise liquidcrystalline) phase. To get an idea about the corresponding range of densities, we note that non-dipolar spherocylinders with $L / D=9$ (which is the largest aspect ratio considered here) order nematically at $\eta \approx 0.25 .^{21}$ The addition of a central longitudinal dipole to the spherocylinders reduces the stability of the nematic phase in favor of the smectic-A phase, while the density at which the transition from the isotropic to the ordered phase occurs does not change too much. ${ }^{16,17}$ Here we perform simulations at $\eta=0.01$ and 0.05 , which should be well within the isotropic regime. In some cases simulations were carried out at a higher volume fraction $(\eta=0.2)$, which is still lower than the onset of the transition to a nematic phase, to probe for the density dependence of the system's behavior.

We use here the following reduced units: distance $\left(d^{*}=d / D\right)$, time $\left(t^{*}=t / \sqrt{\beta D^{2} \nu}\right)$, energy $\left(E^{*}=\beta E\right)$, temperature $\left(T^{*}=\beta / \beta=\right.$ $1)$, magnetic dipole moment $\left(m^{*}=\sqrt{\beta / D^{3}} m\right)$, and magnetic external field $\left(H^{*}=\sqrt{\beta D^{3}} H\right)$, where $\beta=\left(k_{\mathrm{B}} T\right)^{-1}$ and $\nu$ is the mass of the $L^{*}=L / D=9$ spherocylinder (the mass density for all the particles was equal). Note that we have chosen to normalize the energies by $\beta$ instead of the energy parameter $\varepsilon^{-1}$, appearing in the short range potential (1). This choice was made as the physical interpretation of $\varepsilon$ in colloidal particles is not clear. We also note that $m^{*^{2}}=\lambda$ and $m^{*} H^{*}=\xi$ are the commonly used dimensionless parameters measuring the coupling between the particles and with the external field respectively. ${ }^{28}$ For the sake of simplicity we will omit the asterisk of the reduced variables from now on, so for example the aspect ratio will be denoted just by $L$.

Three aspect ratios for the spherocylinders were considered in this study: $L=0$ (spheres), $L=3$ and $L=9$. Concerning the interaction strength we consider two regimes: a high coupling regime in which the particles form aggregates $(m \geq 2$ in the spherical case) and a low coupling regime in which almost no aggregation is observed at the low densities considered here. ${ }^{29}$ To characterize these regimes we employ the degree of polymerization, defined by ${ }^{30,31}$

$$
\Phi=\left\langle\frac{N_{\mathrm{cl}}}{N}\right\rangle,
$$

where $N_{\mathrm{cl}}$ is the number of particles that belong to a cluster and $N$ is the total number of particles. Further, the brackets $\langle\cdots\rangle$ stand for an average in the canonical ensemble, that is,

$$
\langle\cdots\rangle=Z^{-1} \int \cdots \mathrm{e}^{-\beta H} \mathrm{~d} \Gamma
$$

where $Z$ is the canonical partition function and $I$ represents the canonical coordinates and momenta of the system. For the sake of simplicity, the clusters are defined by a simple proximity criterion, ${ }^{3}$ in which two particles are considered to be clustered if the minimum distance between their surfaces is less than $0.1 D$.

Specifically, we choose for the spherical case the values $m=$ 1.5 for the low coupling regime (corresponding to $\Phi<0.1$ ) and $m=2.4$ for the high coupling case $(\Phi>0.9)$. However, using the same values of $m$ for the elongated particles we do not find the required clustering properties, the reason being that the effective dipolar coupling strength in rods is reduced due to their shape (this holds especially in the head-to-tail configuration). Therefore we use here a different criterion to choose $m$ such that the spherocylinders display strong agglomeration in the high coupling regime and almost none at low coupling. Specifically, we followed the approach suggested in ref. 32 in the context of a related model of dipolar rods. The criterion consists in choosing the value for the dipole moment $m^{\prime}$ such that the interaction strength between two adjacent, parallel single dipole rods is the same as that between two parallel rods containing $L+1$ longitudinal dipoles $m$. Using this criterion we find the values $m^{\prime}=2.29$ (low coupling) and $m^{\prime}=3.66$ (high coupling) for the $L=3$ case, and $m^{\prime}=2.8$ (low coupling) and $m^{\prime}=4.48$ (high coupling) for the $L=9$ case.

\section{Results}

\subsection{Structural properties}

Before analyzing the dynamic behavior we will present in this section results pertaining to the structure formation in the systems. This structural analysis complements and helps us to understand part of the dynamic behavior and orientational order presented in later sections.

At the low densities considered here and in the absence of an external field $(\boldsymbol{H}=0)$ the low coupling regime is characterized by a homogeneous distribution of the magnetic particles, independently of the aspect ratio. In the high coupling regime, the systems of magnetic particles display strong aggregation into clusters. However, the nature of such clusters does depend on the aspect ratio. For spherical magnetic particles it is well known that the aggregates formed correspond to head-to-tail chain structures, ${ }^{5,29,33}$ for an illustration see Fig. 2(a). For magnetic rods we rather observe the formation of compact clusters of parallel rods ${ }^{32}$ (as visible in the inset of Fig. 2(b)). We note here again that our definition of high and low coupling regimes stems from these aggregation behavior.
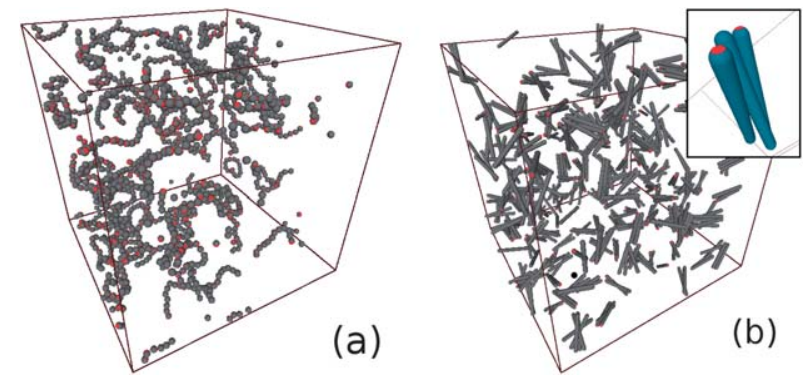

Fig. 2 Configuration at $\eta=0.01$ of (a) dipolar spheres $(L=0, m=2.4)$ and (b) dipolar spherocylinders $(L=9, m=4.8)$, in the absence of an external field $(\boldsymbol{H}=0)$. 

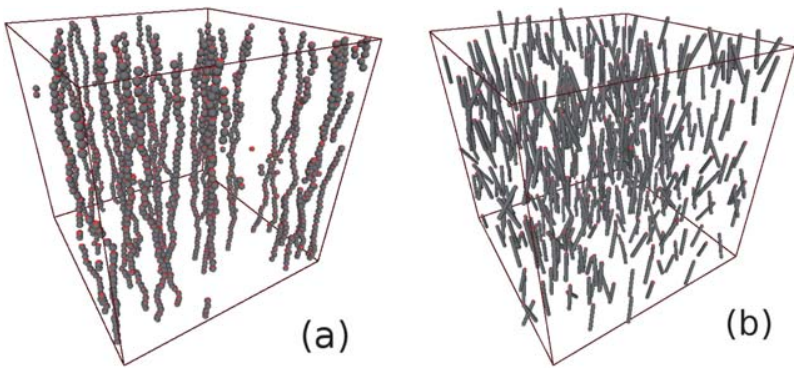

Fig. 3 Configuration at $\eta=0.01$ of (a) dipolar spheres $(L=0, m=2.4)$ and (b) dipolar spherocylinders $(L=9, m=4.8)$, in the presence of an external field $(\boldsymbol{H}=5)$.

In the presence of a relatively strong field $(\boldsymbol{H}=5)$ the spherical magnetic particles form chains that align with the external field ${ }^{34,35}$ (see Fig. 3(a)). This chaining can be further analyzed via suitable projections of the correlation function

$$
h\left(\boldsymbol{r}_{12}, \Omega_{1}, \Omega_{2}\right)=\sum_{l} \sum_{m} \sum_{n} h^{l m n}\left(r_{12}\right) \hat{\Phi}_{00}^{l m n}\left(\hat{\boldsymbol{r}}_{12}, \Omega_{1}, \Omega_{2}\right) .
$$

expanded in terms of rotational invariants $\left(\hat{\Phi}_{\mu \nu}^{l m n}\right) \cdot{ }^{36}$ Of particular importance in our analysis is the projection ${ }^{14}$

$$
\frac{2}{3} h^{112}\left(r_{12}\right)=\frac{1}{(4 \pi)^{2}} \int h\left(\boldsymbol{r}_{12}, \Omega_{1}, \Omega_{2}\right) \hat{\Phi}_{00}^{112}\left(\hat{\boldsymbol{r}}_{12}, \Omega_{1}, \Omega_{2}\right) \mathrm{d} \Omega_{1} \mathrm{~d} \Omega_{2},
$$

where

$$
\hat{\Phi}_{00}^{112}\left(\hat{\boldsymbol{r}}_{12}, \Omega_{1}, \Omega_{2}\right)=3\left(\hat{\boldsymbol{m}}_{1} \cdot \hat{\boldsymbol{r}}_{12}\right)\left(\hat{\boldsymbol{m}}_{2} \cdot \hat{\boldsymbol{r}}_{12}\right)-\hat{\boldsymbol{m}}_{1} \cdot \hat{\boldsymbol{m}}_{2} .
$$

When the particles are oriented by an external field the projection $h^{112}(r)$ is positive for parallel dipoles in a head-to-tail configuration, and negative when they are side by side. Thus it allows us to differentiate between chain formation and parallel configurations.

The formation of chains is signaled by the pair correlation function as a series of peaks separated by a distance of roughly the diameter $D$. As an example, the inset of Fig. 4 shows $h^{112}$ for dipolar spheres in the low and high coupling regimes under the effect of an external field. For the case $m=2.4$ chain

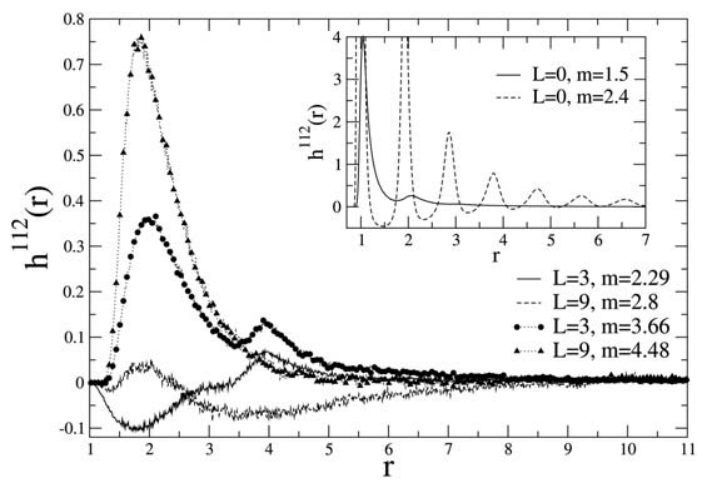

Fig. $4 h^{112}(r)$ function for dipolar spherocylinders under the effect of an external field $(\boldsymbol{H}=5, \eta=0.05)$. The spherical case is shown in the inset. formation is evident from snapshots, and $h^{112}$ presents clear positive peaks at roughly integer multiples of $D$ and negative valleys in between. In the case $m=1.5$ the formation of chains is not evident from the configurations because at this coupling the clusters do not last long, however the $h^{112}$ function presents a couple of peaks at $r=1$ and $r=2$, signaling at the formation of small (less than 4 particles) chains, which are not as stable as the ones formed at high coupling. For the cases $L$ $=3(m=3.66)$ and $L=9(m=4.48)$ we observe that the external field dissolves the aggregates formed by the magnetic rods, as the particles are forced to align their dipole moments with the external field (see Fig. 3(b)). This effect can be quantified by looking at the polymerization degree $(\boldsymbol{\Phi})$. Numerical data for $\boldsymbol{\Phi} v$ s. $\boldsymbol{H}$ are presented in Table 1, where it is observed that in the spherical case the polymerization degree increases as the external field strength is increased, while in the case of the spherocylinders the external field hinders the formation of aggregates.

Dipolar rod systems at high enough values of both $\boldsymbol{H}$ and $m$ may present the formation of head-to-tail alignments in the direction of the field. The plots of $h^{112}(r)$ in Fig. 4 for rods with $L$ $=3$ at high coupling $(m=3.66)$ show two clear positive peaks located at $r \simeq 2$ and $r \simeq 4$. The peak at $r \simeq 4$ corresponds to head-to-tail configurations (Fig. 5(a)), while the one at $r \simeq 2$ corresponds to parallel but shifted configurations like the one shown in Fig. 5(b). In the $L=9$ case there is a single peak at $r \simeq$ 2 that corresponds to shifted configurations like the ones observed in the $L=3$ case. However, no evidence of head-to-tail configuration was observed in the correlation functions or the snapshots for this last case, meaning that the dipole moment is still not strong enough to form chains of rods with such a relatively large aspect ratio.

Table 1 Degree of polymerization as a function of $\boldsymbol{H}$ in the high coupling regime at $\eta=0.01$

\begin{tabular}{llll}
\hline & \multicolumn{1}{l}{} & \\
\cline { 2 - 4 }$H$ & $L=0$ & $L=3$ & $L=9$ \\
\hline 0.0 & $0.939(3)$ & $0.355(4)$ & $0.920(2)$ \\
0.1 & $0.942(2)$ & $0.376(5)$ & $0.936(3)$ \\
0.5 & $0.962(2)$ & $0.255(4)$ & $0.907(2)$ \\
1.0 & $0.971(2)$ & $0.077(2)$ & $0.772(3)$ \\
5.0 & $0.985(1)$ & $0.040(2)$ & $0.039(2)$
\end{tabular}

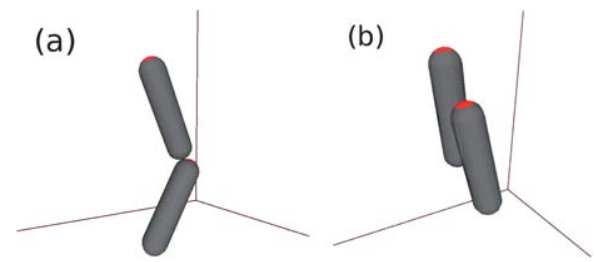

Fig. 5 Cluster of dipolar spherocylinders at $\eta=0.05$ with aspect ratio $L=3, m=$ 3.66 and $\boldsymbol{H}=5$. (a) Head-to-tail configuration. (b) Shifted configuration. 


\subsection{Orientational order}

In this section we investigate how the external field affects the global orientational order of the system, as measured by the order parameter $G_{1}$

$$
G_{1}=\left\langle\frac{1}{N}\left|\sum_{i}^{N} \hat{\boldsymbol{m}}_{i} \cdot \hat{\boldsymbol{d}}\right|\right\rangle .
$$

In eqn (13) $\hat{d}$ denotes the unit eigenvector associated with the largest eigenvalue of the matrix

$$
Q_{k l}=\frac{1}{2 N} \sum_{i=1}^{N}\left(3 \hat{m}_{k}{ }^{i} \hat{m}_{l}{ }^{i}-\delta_{k l}\right),
$$

where $i$ denotes the particle and the indexes $k$ and $l$ denote the cartesian component of the orientation vector.

The orientational order parameter is plotted in Fig. $6(\mathrm{a}-\mathrm{c})$ as a function of the strength of the external field.

We can see that the spheres and the spherocylinders behave differently. As the dipole moment is increased, the dipolar spheres become more susceptible to the external field, resulting in higher values of $G_{1}$. This is not so for the spherocylinders. Already for particles of aspect ratio $L=3$ we see that the curves for the two different values of $m$ are closer to each other as compared to the spherical case. For the longest rods $(L=9)$ the behavior inverses: the system with a lower $m$ value is more susceptible to the external field than the strongly coupled system, except for the highest value of $\boldsymbol{H}$.

The explanation of the phenomena just presented comes from the clustering behavior of the particles. As discussed in Section 3.1, dipolar spheres tend to form chain-like clusters which, under the presence of a homogeneous constant field, align in the direction of the field, and the effect is more pronounced as $\boldsymbol{H}$ increases. In the case of dipolar spherocylinders the alignment imposed by the external field competes with the dipole-dipole interactions. As a consequence, the orientational order at high coupling is lowered compared with that at low coupling.

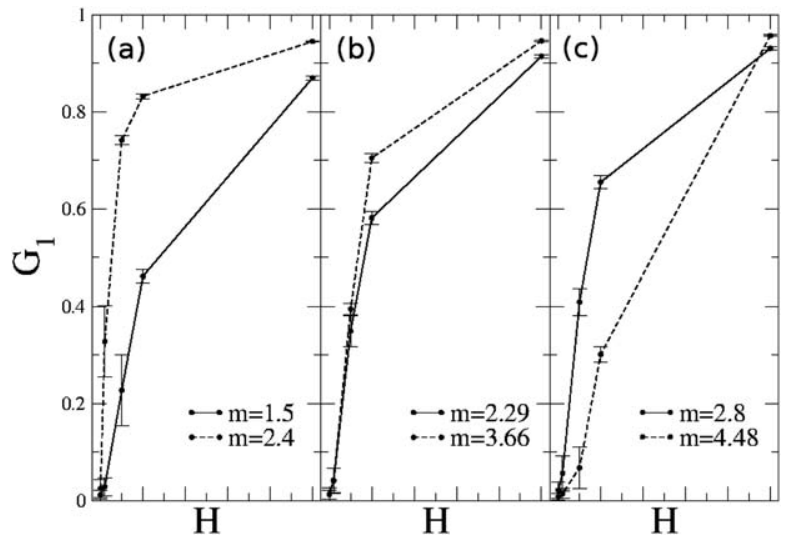

Fig. 6 Orientational order parameter $G_{1}$ as a function of $\boldsymbol{H}$ with $\eta=0.01$. (a) $L=$ 0 , (b) $L=3$ and (c) $L=9$.

\subsection{Translational dynamics}

We now turn to the discussion of the single-particle translational diffusion properties. For systems of magnetic nanospheres, it has been observed experimentally and in simulations that the dipolar interactions lead to a decrease of the translational diffusion constant as compared to the predictions of the Stokes-Einstein theory for non-dipolar spheres. ${ }^{37,38}$ Further, simulations have shown that under the effect of an external field the diffusion coefficients of magnetic spheres in the directions parallel and perpendicular to the field are different (diffusion anisotropy) with the degree of anisotropy varying with the field strength..$^{35,38}$

In this section we investigate how the translational diffusion is affected by the shape of the particles. We focus on systems at a volume fraction of $\eta=0.05$. Our target quantity is the mean square displacement (MSD) of the particles, which in the diffusive regime obeys Einstein's relation

$$
\left\langle\Delta x_{\alpha}^{2}\right\rangle=2 D_{\alpha} t
$$

in the long time limit, where $\Delta x_{\alpha}$ denotes the $\alpha$ cartesian component of the displacement.

The diffusion coefficients $D_{\alpha}$ can be computed whenever the linear relation (15) holds. However, at the low densities under consideration it may happen that the diffusive regime is not reached within the simulation time $(\Delta t=600)$, and thus the diffusion coefficient cannot be obtained. In the remaining cases, we compute the parallel and perpendicular diffusion coefficients, relative to the direction of the external field $(\boldsymbol{H}=H \hat{z})$, as ${ }^{35,38}$

$$
\begin{gathered}
D_{0}=\frac{1}{3}\left(D_{x}+D_{y}+D_{z}\right), \\
D_{\|}=D_{z} \text { and } \\
D_{\perp}=\frac{1}{2}\left(D_{x}+D_{y}\right),
\end{gathered}
$$

as well as the diffusion anisotropy ratio $A=\left(D_{\|}-D_{\perp}\right) / D_{0}$. The resulting values for the diffusion coefficients are shown in Table 2. In all cases we have checked that the values are consistent with those obtained from a Green-Kubo relation involving the velocity autocorrelation function. ${ }^{39}$

Table 2 Diffusion constant obtained via the Einstein relation. Asterisk (*) indicates that the diffusive regime was not reached within the simulation time

\begin{tabular}{lllllll}
\hline$L$ & $m$ & $H$ & $D_{0}$ & $D_{\|}$ & $D_{\perp}$ & $A$ \\
\hline 0 & 1.5 & 0 & 5.6 & - & - & - \\
& & 5 & 5.5 & 3.2 & 6.7 & -0.64 \\
3 & 2.29 & 0 & 3.6 & - & - & - \\
& & 5 & 4.1 & 5.1 & 3.6 & 0.37 \\
& \multirow{2}{*}{3.66} & 0 & $*$ & - & - & - \\
9 & & 5 & 3.0 & 2.5 & 3.3 & -0.27 \\
& \multirow{2}{*}{2.8} & 0 & 2.2 & - & - & - \\
& & 5 & $*$ & $*$ & 1.9 & $*$ \\
& & 0 & 1.3 & - & - & - \\
& & 5 & 2.1 & 2.6 & 1.8 & 0.38
\end{tabular}


We start by considering the dipolar spheres at low coupling. In this case the average diffusion coefficient $\left(D_{0}\right)$ is almost independent of the external field, consistent with the results of previous works. ${ }^{38}$ Furthermore, the diffusion constant perpendicular to the field is observed to be larger than the parallel one $(A<0)$. In order to better understand this behavior of the anisotropy we look at the results presented in Section 3.1, where it was observed that dipolar spheres with $m=1.5$ under an external field of magnitude $\boldsymbol{H}=5$ have the tendency to form short chains. A simple explanation for the negative diffusion anisotropy is that while forming the head-to-tail alignments the amplitude of the fluctuations of the position of the particles reduced in the direction of the field, compared with the direction perpendicular to the field.

The MSD at high coupling presents a more complicated behavior involving sub- and superdiffusion, produced by the formation of rigid chains. We will not go further into its analysis here, as this has been previously discussed by Jordanovic et al. ${ }^{35}$

For the rods we observe in most cases that $D_{\|}>D_{\perp}$, consistent with previous findings in systems of oriented rods. ${ }^{\mathbf{4 0 - 4 2}}$ However, the system of rods with $L=3$ and $m=3.66$ is an exception. Here we find a negative value for the anisotropy. Indeed, we observed in Section 3.1 that in this case the dipolar rods can also form small, loose chains, which can reduce the diffusion in the direction parallel to the field. In contrast, the case $L=9, m=4.48$ does not present such chain formation and its diffusion anisotropy is positive.

\subsection{Rotational dynamics}

In the present section we explore the effects of the dipole-dipole interaction and the external field on the rotational dynamics of individual particles.

We focus on systems at low coupling and in the absence of an external field. In order to see the effect of the dipolar interactions we will compare dipolar spherocylinders with their respective reference systems $(m=0)$. Our target quantity is the single particle time correlation function for the dipole moment (dipole-dipole autocorrelation function)

$$
C_{m}(t)=\frac{1}{3 N}\left\langle\sum_{i=1}^{N} \hat{\boldsymbol{m}}_{i}(t) \cdot \hat{\boldsymbol{m}}_{i}(0)\right\rangle,
$$

which are shown in Fig. 7.

In the case $L=0, m=0$ (see the inset of Fig. 7) the correlation function does not present any decay as there are effectively no angle-dependent interactions. Nevertheless the interaction of the individual dipole moments with the thermostat produces fluctuations around an equilibrium value determined by the kinetic temperature. This changes at $m>0(L=0)$, where we observe a minimum of $C_{m}(t)$ at early times, followed by an increase and a subsequent decay to zero (see the inset of Fig. 7). For rod-like particles, in contrast, the behavior of $C_{m}(t)$ at $m=$ 0 and $m>0$ is very similar. In particular we observe in all cases that $C_{m}(t)$ does not decay monotonically, and there is again a minimum at early times. It is instructive to look additionally at the corresponding frequency dependent response function

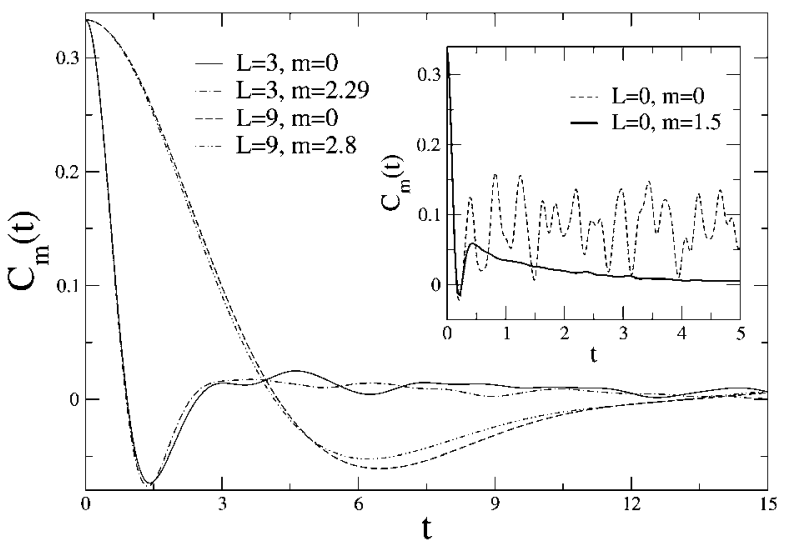

Fig. 7 Single particle dipole-dipole time autocorrelation function at $\eta=0.01$ and $\boldsymbol{H}=0$.

$$
\psi(\omega)=\int_{0}^{\infty}\left(-\frac{\mathrm{d}}{\mathrm{d} t} C_{m}(t)\right) \mathrm{e}^{-i \omega t} \mathrm{~d} t,
$$

whose definition is inspired by that used commonly in linear response theory for the time correlation functions of the magnetization. Results for the imaginary part of $\psi(\omega)$ are plotted in Fig. 8 for the weak coupling case, where the position of the peaks indicates the frequency at which the orientation of the particles is changing.

As the first minimum in the correlations $C_{m}(t)$ is present in systems with and without dipolar interactions at a density in which collisions between particles are not frequent, it could be assumed that this minimum is mostly the product of the inertial rotation of the particles. To check the conjecture about the presence of inertial effects we briefly discuss the rotational motion of a rigid body as described by the Euler equations. ${ }^{43}$ In the spherical case $(L=0)$ and in the absence of external torques the Euler equations reduce to $I \dot{\omega}=0$, where $I$ is the moment of inertia of the sphere and $\omega$ its angular velocity, which is constant. We estimate the rotation frequency of the particles by

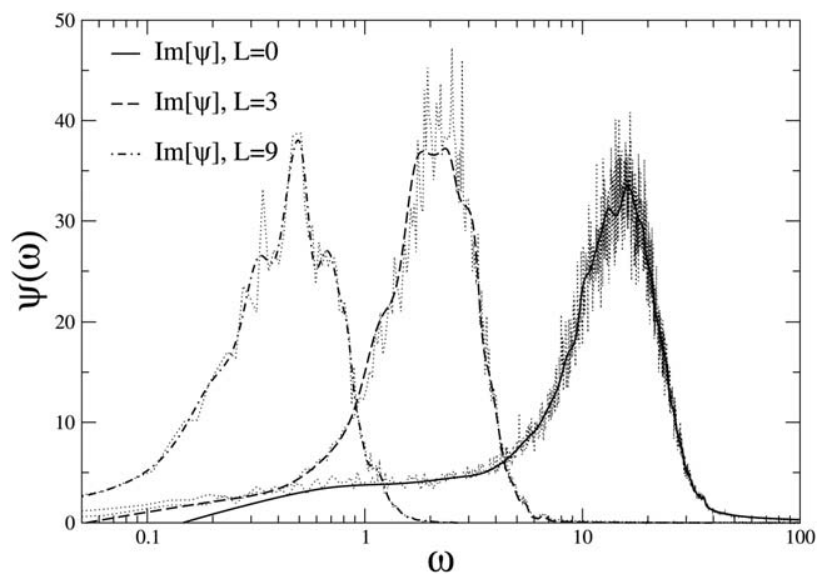

Fig. 8 Frequency response of the particle orientation for dipolar spheres $(m=$ 1.5), spherocylinders with $L=3(m=2.29)$ and spherocylinders with $L=9$ ( $m=$ 2.8). Dotted lines represent raw data. Continuous and dashed lines are spline fittings to the data. 
the corresponding root mean square value in the canonical ensemble. From the equipartition theorem then we obtain $\omega^{\mathrm{rms}}=\left\langle\omega^{2}\right\rangle^{1 / 2}=\sqrt{T / I}=12.04$.

For the case of spherocylinders we compute the root mean square frequency of precession of the non-magnetic rods about the axis parallel to their angular momenta $\left\langle\dot{\phi}^{2}\right\rangle^{1 / 2}$ (see Appendix 5), and obtain the values $\dot{\phi}^{\text {rms }}=2.26$ for $L=3$ and $\dot{\phi}^{\text {rms }}=0.51$ for $L=9$. Indeed, by looking at Fig. 8 it can be seen that the positions of the peaks for the imaginary part of $\psi(\omega)$ are comparable to the values obtained for the root mean square rotation frequency of non-dipolar rods at the low density limit.

In Fig. 9 we present results for $C_{m}(t)$ at higher volume fractions. It is seen that the inertial minimum disappears with increasing density, as the average strength of the interactions increases by virtue of the shorter inter-particle distances. These interactions with neighboring particles produce an effective rotational friction, which in turn "smears out" the inertial effect. Indeed, for a volume fraction of $\eta=0.2$ the decay of $C_{m}(t)$ is already monotonic for systems of spherical particles and spherocylinders $(L=3)$. We note that the results obtained in this section for systems at low density are in agreement with those obtained for the dipole correlation functions using a rotational diffusion model that allows for large angle reorientation of the particles. ${ }^{44}$ The latter model predicts a damped oscillatory behavior for $C_{m}(t)$, rather than a monotonic decay. These considerations show that inertial effects are indeed important at low densities, at least if hydrodynamic interactions are neglected (as is done in our study). However, even in the presence of such interactions, inertial effects could still be relevant. We also note that inertial effects are surely important in the context of molecular liquids. ${ }^{45}$

\subsection{Frequency-dependent magnetic response}

In this section we present results for the collective rotational dynamics. Specifically, we concentrate on the frequencydependent magnetic susceptibility in the low coupling regime. In the framework of linear response theory the change in magnetization $\boldsymbol{M}=\sum_{i} \boldsymbol{m}_{i}$ induced by a weak external field $\boldsymbol{H}(t)=\boldsymbol{H}_{0} \cos \left(\omega_{0} t\right)$ is given by ${ }^{46,47}$

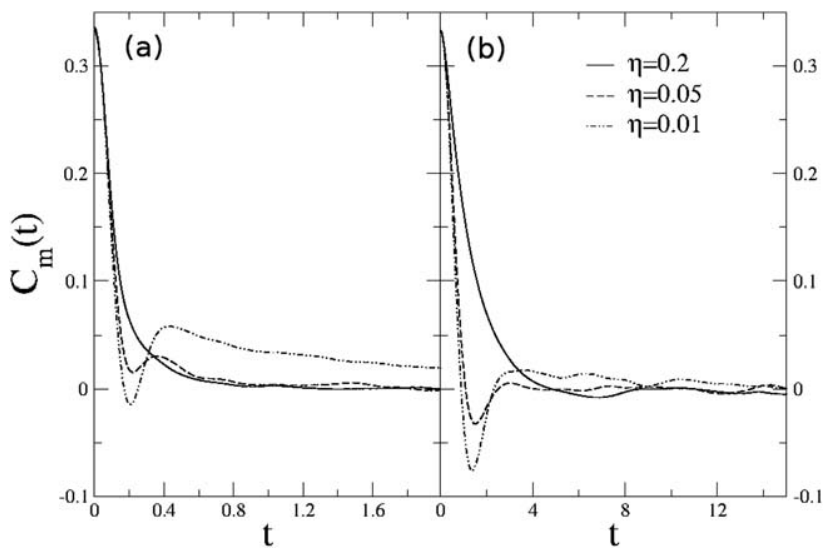

Fig. 9 Dipole-dipole autocorrelation function for several volume fractions. (a) $L$ $=0, m=1.5$. (b) $L=3, m=2.29$.

$$
\langle\Delta M(t)\rangle_{\beta}=-\beta \int_{0}^{\infty}\left(\frac{\mathrm{d}}{\mathrm{d} t^{\prime}}\left\langle M_{\alpha}\left(t^{\prime}\right) M_{\beta}(0)\right\rangle\right) H_{\alpha}\left(t-t^{\prime}\right) \mathrm{d} t^{\prime},
$$

where the sub indexes $\alpha$ and $\beta$ indicate cartesian components.

By Fourier transforming into the frequency domain one obtains

$$
\langle\Delta M(\omega)\rangle_{\beta}=\chi_{\alpha \beta}(\omega) H_{\alpha}(\omega),
$$

with the cartesian components of the susceptibility tensor

$$
\chi_{\alpha \beta}(\omega)=\beta \int_{0}^{\infty}-\left(\frac{\mathrm{d}}{\mathrm{d} t}\left\langle M_{\alpha}(t) M_{\beta}(0)\right\rangle\right) \mathrm{e}^{-i \omega t} \mathrm{~d} t .
$$

As the systems studied here are orientationally disordered at $\boldsymbol{H}=0$, it is sufficient to consider the susceptibility of an isotropic medium,

$$
\chi(\omega)=\beta \int_{0}^{\infty}\left(-\frac{\mathrm{d}}{\mathrm{d} t} C_{M}(t)\right) \mathrm{e}^{-i \omega t} \mathrm{~d} t .
$$

where

$$
C_{M}(t)=\frac{1}{3}\langle\boldsymbol{M}(t) \cdot \boldsymbol{M}(0)\rangle .
$$

In Fig. 10 we plot numerical results for the frequencydependent susceptibility in the low coupling regime at $\eta=$ 0.01 . We first consider the static limit, $\omega \rightarrow 0$. In this limit, the magnetic susceptibility is larger for the magnetic rods than for the magnetic spheres, consistent with the larger slope of the parameter $G_{1}$ as a function of $H$ observed in Fig. 6 . The larger value of $\operatorname{Re}[\chi(\omega \rightarrow 0)]$ is due to the fact that we have employed larger values of $m$ to investigate the low-coupling regime at $L>0$.

Increasing the frequency from zero the functions $\operatorname{Re}[\chi(\omega)]$ first remain positive but then cross zero, followed by a range where they attain negative values. These negative values indicate the presence of oscillations of the magnetisation that are out of phase with the driving field, a behaviour which can also

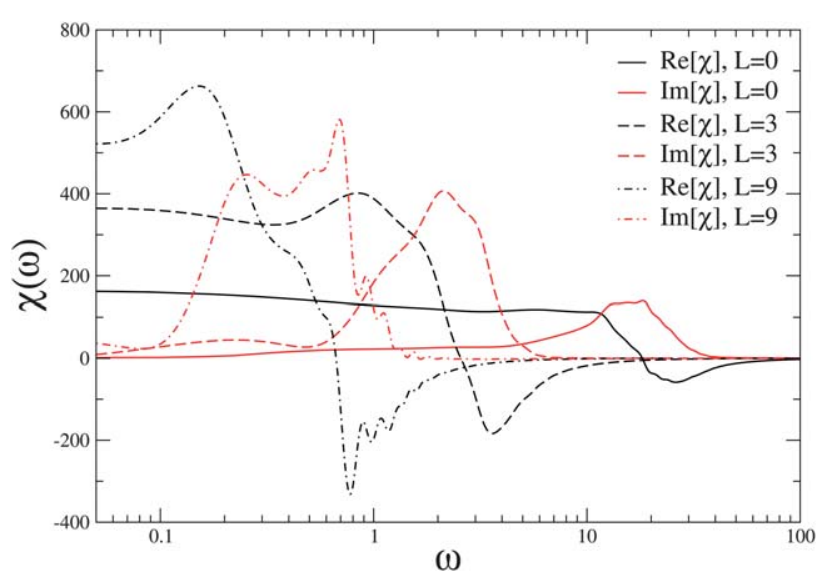

Fig. 10 Frequency-dependent susceptibility for dipolar spheres $(m=1.5)$, spherocylinders with $L=3(m=2.29)$ and spherocylinders with $L=9(m=2.8)$, at $\eta=0.01$. The curves are spline fittings to the data. 
be related to inertial effects. ${ }^{48}$ We also note that the observed behaviour deviates markedly from the Debye-like relaxation seen in systems governed by rotational Brownian motion. Regarding the imaginary part of the susceptibility, it is observed that the absorption peaks in $\operatorname{Im}[\chi(\omega)]$ occur at frequencies that are lower, while the aspect ratio of the particles is larger. Moreover, these peaks are located in the same frequency regions as their single particle counterparts (Fig. 8). Since the latter are dominated by inertial effects we conclude that such effects also determine the collective response at the low density and coupling strength considered.

We now turn to the role of density. To this end we plot in Fig. 11 the correlation function $C_{M}(t)$, which represents the kernel in eqn (24), at several volume fractions. As in the case of single particle rotational dynamics, we observe that by increasing the density inertial effects on the susceptibility become less pronounced. In particular, for magnetic spheres at a relatively high volume fraction $(\eta=0.2)$ the function $C_{M}(t)$ presents a Debye-like behavior in the sense that it can be fitted as having a single decay time (i.e. by a function of the type $\exp (-t / \tau))$. However, at lower densities, there are clear deviations from such behavior. Importantly, this also holds in the case of spherocylinders, where $C_{M}(t)$ is non-exponential at all densities considered.

We look now at the frequency-dependent susceptibility for the systems at higher densities. Fig. 12 plots the real and imaginary parts of $\chi(\omega)$ for systems of spheres and spherocylinders $(L=3)$ in the low coupling regime at three different volume fractions. In both the spherical and spherocylinder cases it is seen that at the lowest volume fraction $(\eta=0.01)$ the absorption peaks in $\operatorname{Im}[\chi(\omega)]$ are located at frequencies close to those of free particle rotation, computed in Section 3.4. As the density increases the peaks widen and shift towards lower frequencies, an effect which can be attributed to the increasingly important role of interactions between particles. However, these collective effects manifest differently in the system of spheres and that of spherocylinders. In the spherical case the height of the absorption peak increases with the density, meaning that the applied oscillating field $\boldsymbol{H}(t)$ needs

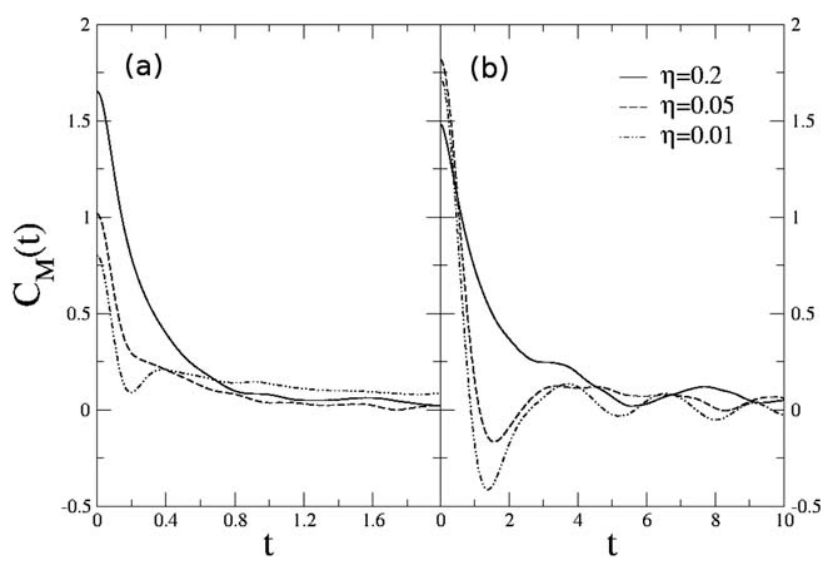

Fig. 11 Autocorrelation functions of the magnetisation for (a) dipolar spheres and (b) spherocylinders with $L=3$ at low coupling.

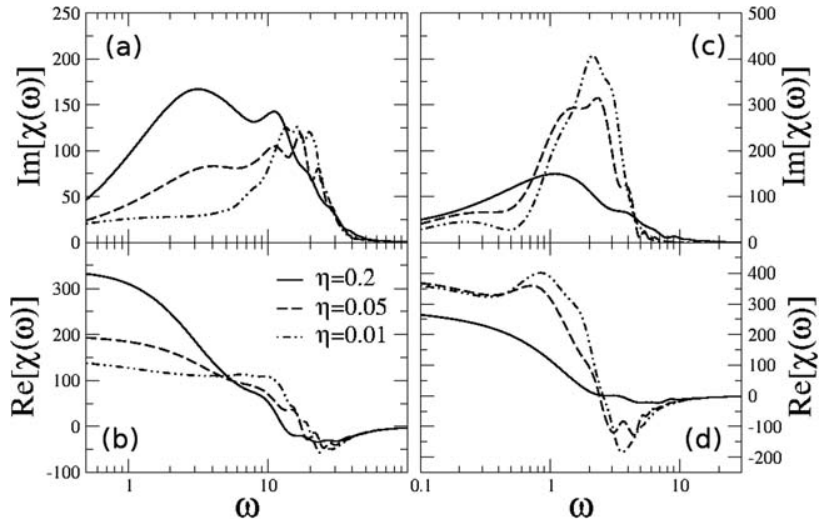

Fig. 12 Frequency dependent susceptibility for dipolar spheres ((a) and (b)) and spherocylinders of aspect ratio $L=3((c)$ and $(d))$, at low coupling. The curves are spline fittings to the data.

to do a higher amount of work $\Delta W(\omega) \propto H_{0}{ }^{2} \operatorname{Im}[\chi(\omega)]$ on the system through an oscillation. ${ }^{49}$ At the same time, the susceptibility of the system in the static limit $\operatorname{Re}[\chi(\omega \rightarrow 0)]$ increases. In the case of the spherocylinder the opposite behavior is observed. As the density is increased the amount of work done by the external field per cycle in the frequency region of the absorption peak as well as the susceptibility in the static limit both decrease. The fundamental difference between the sphere and spherocylinder systems lies in their shape: while steric interactions play no role in the rotational motion of the spheres, the spherocylinders at high densities will collide frequently with each other as they rotate, hindering their capacity to reorient and follow the external field. It is plausible that this reduces the susceptibility of the system.

\section{Summary and conclusions}

In this paper we have explored the structural and dynamical behavior of a model suspension of magnetic nanorods, focusing on densities below the isotropic-nematic transition. Our MD simulation results reveal that the system's behaviour differs markedly from that of magnetic spheres, both with and without a magnetic field. The main results can be summarized as follows:

- In the absence of a field dipolar rods favor compact, sideby-side configurations with antiparallel ordering of the dipole moments. A sufficiently strong external field tends to dissolve these clusters. As a consequence, an increase of the coupling strength $(m)$ yields a decrease of the zero-frequency susceptibility of magnetic rod suspensions. This is in strong contrast to the behaviour of magnetic spheres: here, the particles form chain-like head-to-tail clusters at zero field which are rather stabilised by an external field (yielding an increase of the susceptibility with $m$ ).

- In the presence of a magnetic field, the translational diffusion of magnetic rods becomes anisotropic with respect to the directions parallel and perpendicular to the field. Similar anisotropic behaviour is seen in systems of dipolar spheres. ${ }^{35,38}$ 
However, in contrast to the spherical case the diffusion constant along the field $\left(D_{\|}\right)$is generally larger than the perpendicular one $\left(D_{\perp}\right)$, consistent with the behavior of non-polar, oriented rods. ${ }^{\mathbf{4 0 - 4 2}}$ An exception is a system of relatively short rods $(L=3)$ with strong dipole moments: here, application of a large external field can enforce the particles to form head-totail clusters, yielding a behaviour similar to that of spheres (i.e., $D_{\perp}<D_{\|}$).

- The rotational diffusion of magnetic rods in the absence of an external field and under dilute, low-coupling conditions is dominated by inertial effects. The latter generate a minimum in the time correlation function of the particle orientation $C_{m}(t)$ or, equivalently, a peak in the corresponding Fourier spectrum. The location of this peak can be estimated analytically by considering ensembles of non-interacting particles. By increasing the density, interaction effects between the particles become more relevant. As a result, the effects of inertia are reduced until the decay of $C_{m}(t)$ becomes monotonic for the largest density considered. We note, however, that one would expect additional effects if the density is increased towards values pertaining to a nematic phase.

- For low densities, the behaviour of the collective dipoledipole correlation function and the frequency-dependent susceptibility is similar to that observed in the single-particle dynamics, in that all functions are dominated by inertial effects. At higher densities, the collective response strongly depends on the particle's shape: for spheres, one eventually observes an exponentially decaying correlation function $C_{M}(t)$ characterised by a single relaxation time, leading to a Debye-like response function in the frequency domain. For the dipolar-rod systems, however, the behaviour of $C_{M}(t)$ is more complex at all densities considered, presumably due to the increasing importance of steric interactions.

To conclude, our MD simulation results indicate that, compared to conventional ferrofluid composed of spherical particles, suspensions of magnetic nanorods can display a markedly distinct structural and dynamical behaviour. Clearly, the present study represents just a first step towards a full microscopic understanding of the material properties of such systems; more detailed future investigations should explore a broader region of parameters as well as the role of hydrodynamic interactions. Moreover, given the different behaviour of time correlation functions it seems very interesting to explore the impact of these dynamical features on rheological and transport properties. Work in these directions is in progress.

\section{Appendix A - rotation frequency of torque free spherocylinders}

In this appendix we derive an expression for the root meansquared frequency of rotation for a spherocylinder without external torque. A sketch of the situation is given in Fig. 13. The particle with orthonormal body axes $\left(\hat{\boldsymbol{e}}_{1}, \hat{\boldsymbol{e}}_{2}, \hat{\boldsymbol{e}}_{3}\right)$ spins around its symmetry axis and undergoes precession around the direction of its angular momentum $(\boldsymbol{L})$ at an angle $\theta .{ }^{\mathbf{4 3}}$ Assume now a gas of such non-interacting spherocylinders with diameter $D$,

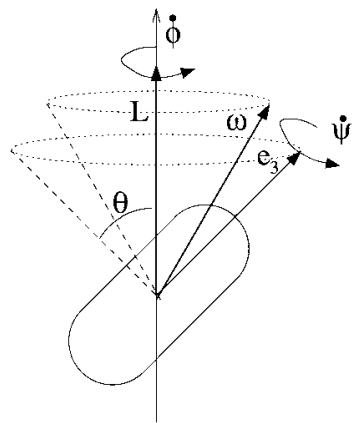

Fig. 13 Sketch of the rotational motion of a spherocylinder in the absence of external torque.

length $L$ and mass density $\rho$ (see Fig. 1). For each particle, the tensor of moments of inertia in the body reference frame is given by

$$
\overline{\boldsymbol{I}}=\left(\begin{array}{ccc}
I_{\perp} & 0 & 0 \\
0 & I_{\perp} & 0 \\
0 & 0 & I_{\|}
\end{array}\right)
$$

where

$$
I_{\|}=\rho \pi\left(\frac{L D^{4}}{32}+\frac{D^{5}}{60}\right)
$$

and

$$
I_{\perp}=\rho \pi\left(\frac{L^{3} D^{2}}{48}+\frac{L^{2} D^{3}}{24}+\frac{L D^{4}}{64}+\frac{D^{5}}{60}\right) .
$$

We now want to obtain the average precession frequency of the spherocylinders when the system is in the canonical ensemble. The squared angular momentum of a symmetric top in terms of the Euler angles $(\phi, \theta, \psi)^{\mathbf{4 3}}$ and their time derivatives is

$$
L_{2}=I_{\perp}{ }^{2}\left(\dot{\theta}^{2}+\dot{\phi}^{2} \sin ^{2} \theta\right)+I_{\|}^{2}(\dot{\phi} \cos \theta+\dot{\psi})^{2} .
$$

We now take $\dot{\theta}=0$ and the relation $\dot{\phi} \cos \theta+\dot{\psi}=\omega_{3}$, where $\omega_{3}$ is the component of the angular velocity parallel to $\hat{\boldsymbol{e}_{3}}\left(L_{3}\right)$ in the body reference frame. This yields

$$
L_{2}=I_{\perp}^{2} \dot{\phi}^{2} \sin ^{2} \theta+I_{\|}^{2} \omega_{3}^{2}
$$

Now we note that

$$
I_{\|}^{2} \omega_{3}^{2}=L_{3}^{2}=\left(\boldsymbol{L} \cdot \hat{\boldsymbol{e}}_{3}\right)^{2}=L_{2} \cos ^{2} \theta .
$$

Combining eqn (30) and (31) we get ${ }^{43}$

$$
\dot{\phi}^{2}=\frac{L^{2}}{I_{\perp}^{2}} \text {. }
$$

Finally we take the average of (32) in the canonical ensemble to obtain

$$
\left\langle\dot{\phi}^{2}\right\rangle=\frac{I_{\perp}{ }^{2}\left\langle\omega_{1}{ }^{2}\right\rangle+I_{\perp}{ }^{2}\left\langle\omega_{2}{ }^{2}\right\rangle+I_{\|}{ }^{2}\left\langle\omega_{3}{ }^{2}\right\rangle}{I_{\perp}{ }^{2}}=\frac{2 I_{\perp}+I_{\|}}{I_{\perp}{ }^{2}} k_{\mathrm{B}} T
$$




\section{Acknowledgements}

We gratefully acknowledge financial support from the Deutsche Forschungsgemeinschaft through grant KL1215/7.

\section{References}

1 L. Vékás, Adv. Sci. Tech., 2008, 54, 127.

2 C. Scherer and A. M. Figueiredo Neto, Braz. J. Phys., 2005, 35, 718.

3 C. Holm and J.-J. Weis, Curr. Opin. Colloid Interface Sci., 2005, 10, 133.

4 M. R. Jolly, J. W. Bender and J. D. Carlson, J. Intell. Mater. Syst. Struct., 1999, 10, 5.

5 P. Domínguez-Garcíaa and M. A. Rubio, Colloids Surf., A, 2010, 358, 21.

6 J. P. Lu, Phys. Rev. Lett., 1995, 74, 1123.

7 S. Lian, E. Wang, Z. Kang, L. Bai, Y. Gao, M. Jiang, C. Hu and L. Xu, Solid State Commun., 2004, 129, 485.

8 A. Gole, J. W. Stone, W. R. Gemmill, H.-C. zur Loye and C. J. Murphy, Langmuir, 2008, 24, 6232.

9 A. Günther, P. Bender, A. Tschöpe and R. Birringer, J. Phys.: Condens. Matter, 2011, 23, 325103.

10 R. Birringer, H. Wolf, C. Lang, A. Tschöpe and A. Michels, Z. Phys. Chem., 2008, 222, 229.

11 B. J. Lemaire, P. Davidson, J. Ferré, J. P. Jamet, P. Panine, I. Dozov and J. P. Jolivet, Phys. Rev. Lett., 2002, 88, 125507.

12 J. Philip, P. D. Shima and B. Raj, Appl. Phys. Lett., 2007, 91, 203108.

13 C. P. Bean, J. Appl. Phys., 1955, 26, 1381.

14 G. J. Zarragoicoechea, J.-J. Weis and D. Levesque, Mol. Phys., 1991, 74, 629.

15 B. Groh and S. Dietrich, Phys. Rev. E: Stat. Phys., Plasmas, Fluids, Relat. Interdiscip. Top., 1997, 5, 2892.

16 M. Houssa, L. F. Rull and S. C. McGrother, J. Chem. Phys., 1998, 109, 9529.

17 A. Gil-Villegas, G. Jackson and S. C. McGrother, J. Mol. Liq., 1998, 76, 171.

18 J. S. van Duijneveldt, A. Gil-Villegas, G. Jackson and M. P. Allen, J. Chem. Phys., 2000, 112, 9092.

19 M. Houssa, A. Oualid and L. F. Rull, Mol. Phys., 1998, 94, 439. 20 K. Satoh, Mol. Cryst. Liq. Cryst., 2008, 480, 202.

21 P. Bolhuis and D. Frenkel, J. Chem. Phys., 1997, 106, 666.

22 W.-L. Bao, L. Low, J. Jiang and J. Y. Ying, J. Mater. Chem., 2012, 22, 7117.

23 S. Odenbach and S. Odenbach, Ferrofluids: Magnetically Controllable Fluids and their Applications, Springer, Berlin, 2002.
24 K. Watanabe, H. Okajima, T. Kato and H. Hamaguchi, J. Chem. Phys., 2012, 136, 014508.

25 M. Allen and D. J. Tildesley, Computer Simulation of Liquids, Clarendon Press, Oxford, 1991.

26 M. Schoen, S. H. L. Klapp, K. B. Lipkowitz, T. R. Cundari and D. B. Boyd, Reviews in Computational Chemestry: v.24, WileyBlackwell, Oxford, 2007.

27 B. Leimkuhler, E. Noorizadeh and F. Theil, J. Stat. Phys., 2009, 135, 261.

28 M. I. Shliomis, Phys.-Usp., 1974, 17, 153.

29 J.-J. Weis and D. Levesque, Phys. Rev. Lett., 1993, 71, 2729.

30 S. S. Das, A. P. Andrews and S. C. Greer, J. Chem. Phys., 1995, 102, 2951.

31 H. Schmidle, C. K. Hall, O. D. Velevb and S. H. L. Klapp, Soft Matter, 2012, 8, 1521.

32 C. E. Alvarez and S. H. L. Klapp, Soft Matter, 2012, 8, 7480.

33 K. Butter, P. M. Bomans, P. H. Frederik, G. J. Vroege and A. P. Philipse, J. Phys.: Condens. Matter, 2003, 15, S1451.

34 P. G. de Gennes and P. A. Pincus, Phys. kondens. Mater., 1970, 11, 189.

35 J. Jordanovic, S. Jger and S. H. L. Klapp, Phys. Rev. Lett., 2011, 106, 038301.

36 L. Blum and A. Torruella, J. Chem. Phys., 1972, 56, 303.

37 J. C. Bacri, A. Cebers, A. Bourdon, G. Demouchy, B. M. Heegaard and R. Perzynski, Phys. Rev. Lett., 1995, 74, 5032.

38 P. Ilg and M. Kröger, Phys. Rev. E: Stat., Nonlinear, Soft Matter Phys., 2005, 72, 031504.

39 D. J. Evans and G. Morriss, Statistical Mechanics of Nonequilibrium Liquids, Cambridge University Press, Cambridge, 2008.

40 S. Hess, D. Frenkel and M. P. Allen, Mol. Phys., 1991, 74, 765. 41 S. V. Dvinskikh and I. Furó, J. Chem. Phys., 2001, 115, 1946. 42 P. Ilg, Phys. Rev. E: Stat., Nonlinear, Soft Matter Phys., 2005, 71, 051407.

43 L. D. Landau and E. M. Lifshitz, Mechanics, Pergamon Press, Oxford, 1976.

44 R. G. Gordon, J. Chem. Phys., 1966, 44, 1830.

45 J. Hansen, D. Levesque and J. Zinn-Justin, Liquids, Freezing and Glass Transition, Les Houches, Session LI, North Holland, Amsterdam, 1991.

46 U. M. Titulaer and J. M. Deutch, J. Chem. Phys., 1974, 60, 1502.

47 V. A. Froltsov and S. H. L. Klapp, J. Chem. Phys., 2007, 126, 114703.

48 B. Kevin and P. Scaife, Principles of Dielectrics, Clarendon Press, Oxford, 1998.

49 C. J. F. Böttcher and P. Bordewijk, Theory of Electric Polarization, Elsevier, Amsterdam, 1996, vol. 2. 